Décadrages Décadrages

cinéma, à travers champs Cinéma, à travers champs

$15 \mid 2009$

Raoul Ruiz

\title{
Trois vies et un seul cinéaste (Raoul Ruiz)
}

Des récits singuliers qui se conjuguent au pluriel

\section{Alain Boillat}

\section{OpenEdition}

Journals

Édition électronique

URL : https://journals.openedition.org/decadrages/122

DOI : $10.4000 /$ decadrages.122

ISSN : 2297-5977

Éditeur

Association Décadrages

\section{Édition imprimée}

Date de publication : 1 octobre 2009

Pagination : 8-24

ISBN : 978-2-9700668-0-4

ISSN : 2235-7823

Référence électronique

Alain Boillat, «Trois vies et un seul cinéaste (Raoul Ruiz) », Décadrages [En ligne], 15 | 2009, mis en ligne le 26 novembre 2012, consulté le 03 avril 2022. URL : http://journals.openedition.org/ decadrages/122 ; DOI : https://doi.org/10.4000/decadrages.122 


\title{
Trois vies et un seul cinéaste (Raoul Ruiz). Des récits singuliers qui se conjuguent au pluriel
}

\author{
par Alain Boillat
}

1 Voir notamment l'entretien filmé avec Jérôme Prieur, Raoul Ruiz. Le Voyage extraordinaire, 2006. Cette démarche consonne avec celle de René Clair (Paris qui dort, Le Voyage imaginaire,...) ou d'autres cinéastes associés au "cinéma impressionniste" français des années 1920 (Dulac, L'Herbier, etc.). Une telle parenté avec une esthétique du "muet" explique l'énorme liberté avec laquelle Ruiz traite les rapports entre les images et la piste son (à l'instar de René Clair dans ses films "parlants").

2 Raoul Ruiz, "Théorie du conflit central", dans Poétique du Cinéma 1 (Miscellanées), Editions Dis Voir, Paris, 1995.
L'œuvre de Raoul Ruiz, que nous proposons d'envisager ici dans une optique narratologique à travers quelques exemples emblématiques, tend à se soustraire à une analyse de détail en raison du principe de prolifération qui la gagne (et la travaille avec une productivité certaine) à divers niveaux. En effet, non seulement le cinéaste est extrêmement prolifique, mais chacun de ses films, produit d'un pouvoir de fabulation proprement inouï, est composé d'une multitude d'histoires dont les fils se croisent et qui se génèrent mutuellement selon un processus d'associations libres. L'«esprit de bricolage» que Raoul Ruiz aime à prôner sur le plan technique, dans une démarche de réhabilitation d'un fantastique artisanal à la Méliès ${ }^{\mathbf{1}}$, concerne certes prioritairement la composition d'une image qui ne craint pas de provoquer d'ostentatoires distorsions du réel que d'aucuns pourraient taxer de "gratuites»- on verra que le cinéaste vise précisément cette gratuité en abolissant les règles de causalité. Toutefois, on peut considérer qu'il porte également sur la construction même des récits, dont le caractère hétéroclite et la conduite désinvolte se situent aux antipodes des modèles de linéarité et de cohérence qui prévalent dans le cinéma dominant.

Contrairement à une certaine modernité cinématographique qui s'est vouée à l'exploration de l'en deçà du Narratif, Ruiz s'adonne, de façon spontanée et viscérale, à l'excès inverse : il s'agit pour lui de créer un foisonnement dont les modalités de déploiement s'accordent à l'ambiguïté inhérente au genre fantastique qu'il affectionne tout particulièrement. Etant donné la singularité des partis pris ruiziens quant à l'agencement du récit, nous considérerons dans le premier temps de cette étude l'un des textes théoriques du cinéaste que nous mettrons en rapport avec sa pratique: il s'agit de l'essai intitulé «Théorie du conflit central», version écrite d'une conférence donnée en avril 1994 à l'Université de Duke aux Etats-Unis et qui a paru l'année suivante en français dans Poétique du cinéma ${ }^{2}$. Inscrivant ses films dans la filiation des récits 
picaresques, gigognes ou digressifs de la littérature anglaise ou hispanophone (Miguel de Cervantes, Baltasar Gracián, Laurence Sterne, le Stevenson de l'Ile au trésor [1883] ou du Dynamiteur [1885]) 3, Ruiz se soustrait au modèle de la narration "classique» bien plus qu'il n'entend en "déconstruire les codes", comme se sont plu à le faire certains tenants de la modernité. C'est pourquoi son pamphlet contre la forme classique - qu'il s'agit d'appréhender, tout comme ses films, dans sa dimension ironique et ludique - ressortit de l'essai de poétique, non de la proposition d'un modèle qu'il s'agirait d'appliquer. Bien que la réflexion du cinéaste ne s'appuie aucunement sur la tradition de la narratologie française, il nous paraît néanmoins productif de l'envisager à l'aune de ce cadre théorique pour en dégager parentés et singularités.

\section{Une diatribe contre la narration "classique"}

L'article "Théorie du conflit central», qui constitue l'un des sept chapitres du premier tome de Poétique du cinéma, met d'entrée de jeu l'accent sur la notion que l'auteur s'emploiera à condamner. Avec ses nombreuses digressions, ses références érudites tous azimuts qui convoquent divers champs du savoir et une argumentation tributaire de l'humeur du conférencier, ce texte n'est pas sans ressembler, dans sa forme même, à la structure hétérogène des films de Ruiz et aux sinuosités de leur récit ${ }^{4}$. Or le discours que le réalisateur y développe est riche en enseignements sur sa poétique, elle-même sous-tendue par une vision du monde ouverte à toutes les virtualités, totalement étrangère à l'idée d'une régulation ou d'une normativisation, la narration relevant chez lui du pulsionnel. Le cinéaste s'oppose à la nécessité, relevée par la quasi-intégralité des manuels sur le scénario qui s'inspirent invariablement des préceptes aristotéliciens appliqués à la tragédie $\mathbf{5}$, d'organiser le récit autour d'un "conflit central» - c'est-à-dire, en termes greimassiens, autour de la présence d'un actant-sujet désirant atteindre un but et se confrontant dans cette quête à des opposants ${ }^{6}$. Le cinéaste sape ainsi l'instrument clé de réduction des récits à leur noyau, que cela soit dans l'optique des sémanticiens (qui isolent et combinent des constituants minimaux) ou dans celle des partisans de l'efficience scénaristique (dont le format réduit du pitch est emblématique). Les récits de Ruiz sont totalement étrangers au modèle d'Aristote. Il n'y a pas un centre (de l'intrigue), mais plusieurs ; au vu de leur profusion, cela revient à dire qu'il n'y a pas de centre du tout. Le refus du conflit en tant qu'élément structurant a des incidences idéologiques, puisqu'il interdit tout manichéisme et permet de proposer un cinéma de l’ambiguïté. Les récits ruiziens - car, précisons-le, le (méta) narratif demeure central dans les films du cinéaste - ne se présentent en
3 La plupart de ces références sont significativement antérieures au roman réaliste ou naturaliste du XIXe siècle qui constitua l'objet privilégié de la narratologie structuraliste.

4 Dans l'avant-propos de l'ouvrage, Ruiz explique ainsi sa démarche: "J'ai choisi un genre proche de ce qu'on appelait, dans l'Espagne du XVIe siècle, les Miscelaneas (Miscellanées), des discours théorico-narratifs où I'on s'exerce à faire des cabrioles, des changements de cap inattendus, des interpolations extravagantes, bref ce que l'on pourrait appeler "l'art de passer du coq à l'âne ". (Poétique du cinéma 1, op. cit., pp. 7-8).

5 Ruiz mentionne dans son texte l'ouvrage Comment écrire un script de Lawson, mais ses remarques sont valables pour tous les ouvrage de ce type (ceux de Robert McKee, Syd Field, Christian Biegalski, etc.) qui, depuis la fin des années 1970, connaissent pour certains un succès retentissant et constituent une véritable manne pour les éditeurs. La popularité de tels manuels n'est peut-être pas sans lien avec le constat de Ruiz: "Il y a trente ou quarante ans, cette théorie était un mode d'emploi facultatif à l'usage des principaux fabricants de l'industrie cinématographique américaine. Aujourd'hui, c'est une loi" ("Théorie du conflit central ", op. cit., p. 9).

6 Notons que la sémantique narrative de Greimas est souvent sous-jacente aux conceptualisations proposées dans ces manuels, qui se gardent toutefois le plus souvent d'y faire explicitement référence. 
7 Voir notamment David Bordwell et alii, The Classical Hollywood Cinema, Columbia University Press, New York, 1985, pp. 12-23.

8 Raoul Ruiz, "Théorie du conflit central", op. cit., p. 10.

9 Philippe Hamon, "Un discours contraint", dans R. Barthes et alii, Littérature et réalité, Seuil (coll. Points Essais), Paris, 1982, p. 134.

10 Selon la traduction de J. Hardy [1932], Poétique, Gallimard, Paris, 1996, p. 90. effet pas comme une charpente unique, mais comme le résultat d'une constante expansion, d'une juxtaposition d'éléments qui ne sont pas hiérarchisés entre eux, mais s'appellent l'un l'autre, se font écho. C'est pourquoi les répliques des personnages s'organisent parfois selon un principe plus énumératif que dialogique ou narratif pour rendre compte du bric-à-brac des univers décrits. Ainsi, dans Les Trois Couronnes $d u$ matelot (France/Chili, 1982), la sœur du marin mentionne lors du départ de celui-ci l'ensemble des présents qu'elle aimerait qu'il lui rapporte (bague, collier, bicyclette, café), tandis que, plus tard, le personnage de Maria inventorie un grand nombre d'objets susceptibles d'être comparés au cercueil (une brosse à dents, une voiture, etc.) qui trône dans sa chambre à coucher.

Dans sa conférence, Ruiz mine tous les principes établis par David Bordwell dans son évocation des caractéristiques majeures du cinéma «classique hollywoodien » $\mathbf{7}$ : dès lors que l'intentionnalité du sujet (et, comme nous le verrons, son unité) est remise en cause, la sacro-sainte causalité sur laquelle repose la vraisemblance du récit passe à la trappe (au sens d'une sorte de "truc", dans un rapport ludique au spectateur). Les films deviennent ainsi, selon les termes du cinéaste, "aussi invraisemblables et extravagants que la vie elle-même» $\mathbf{8}$, comme s'il s'agissait de prendre acte du dicton selon lequel "la réalité dépasse la fiction" pour revigorer cette dernière. Comme l'ont montré les théoriciens de la littérature, le réalisme - ou «l'effet de réel», pour reprendre la terminologie de Barthes - est avant tout produit par la cohérence interne de l'œuvre assurée par la motivation logique de chacune des composantes du récit. Philippe Hamon avançait à la suite de Jakobson qu' «il semblerait donc que, pour le lecteur (comme pour le spectateur de tableau ou de spectacle), le réel soit d'abord le cohérent» 9 . La plupart des narratologues ont établi les conditions minimales de l'intrigue en respectant la définition aristotélicienne de l'étendue de la fable, soit "une suite d'événements, qui se succèdent suivant la vraisemblance ou la nécessité, [permettant] de faire passer le héros de l'infortune au bonheur ou du bonheur à l'infortune $\stackrel{\mathbf{1 0}}{ }$.

Chantre de l'incohérence, Ruiz enraie quant à lui tout effet de réel : que ses films relèvent du genre fantastique ou non (notion qui a trait à la nature du représenté, alors que Ruiz s'attaque à la représentation même en reversant l'étrangeté provoquée par sa déconstruction narrative sur le monde du film), ils ne mettent pas en place, pour la plupart, un univers réaliste. Dans l'argumentaire de son article, le cinéaste mobilise également une dimension autobiographique - qui se traduit, chez cet exilé qui se réinvente continuellement un passé (en multipliant, là aussi, les 
possibles) par une dimension "autofictionnelle»: ses études en cinéma l'auraient conduit à "découvrir que tous les films américains étaient soumis à un système de crédibilité»11, et que ce primat de la vraisemblance impliquait également un système de valeurs et une normativité (les films dits "bien faits» du point de vue narratif) qui contredisaient totalement sa passion d'adolescent pour les séries B vues dans les salles chiliennes 12. Il précise à propos de ces films que «beaucoup étaient sans queue ni tête, monstrueux peut-être par surabondance de queues et de têtes»13. La monstruosité qu'il évoque ici correspond tout à fait à ce qu'il cherche à faire advenir dans ses propres films - non dépourvus d'une dimension feuilletonesque et «sérielle»-, où il ne s'agit pas d'aller d'un point $A$ à un point $B$ (en affrontant des protagonistes hostiles ou en surmontant des obstacles), mais de multiplier ces points - qui sont aussi des "points de vue» - dans une cartographie fantasmatique qui favorise la répétition, la digression, l'aller-retour et le palimpseste du surplace, c'est-à-dire une forme où les versions du «réel» se superposent sans s'exclure, faisant partie intégrante d'un même monde composé de diverses facettes. Ruiz est tenté par une conception cyclique du Narratif qu'il exprime notamment via la mise en scène de pratiques ritualisées (mythes, folklore, légendes marines, dispositif du «tableau vivant», etc.) suggérant que tout a déjà été dit. Ainsi, l'interlocuteur du narrateur des Trois couronnes $d u$ matelot ne cesse d'affirmer à ce dernier qu'il a déjà entendu l'histoire (pourtant totalement insolite) qu'il lui rapporte, et, à la fin du film, le frappe à mort en se montrant indigné du caractère abject d'un épisode de l'histoire (la contamination des corps par des vers) à propos duquel il avait justement dit qu'il s'agissait du seul passage inédit. Pointant le rayonnage d'une bibliothèque, un garçon déclare par ailleurs au matelot que "tous ces écrivains ont déjà écrit [son] histoire plusieurs fois". Totalement dénué de libre-arbitre, le personnage ruizien est l'expression désenchantée d'un devenir fantomatique de l'humain. La conception d'un ressassement de motifs contenus dans une sorte de bibliothèque borgésienne est également partagée par l'un des psychanalystes de Généalogie d'un crime (France/Portugal, 1997), qui identifie dans les meurtres la répétition inconsciente de scènes narrées par des textes littéraires (pour Ruiz, la psyché n'est autre qu'un univers de fiction). Même lorsqu'il ne s'agit pas d'«adaptations» (toujours «libres»), la culture livresque du cinéaste est explicitement imbriquée dans la diégèse de ses films.

En dépit de la répétitivité des récits, les conteurs ruiziens s'adonnent à leur activité de façon jubilatoire et obsessionnelle, fascinés par la séduction de la fable et peut-être aussi par son inanité même. A force de
11 Raoul Ruiz, "Théorie du conflit central", op. cit., p. 11.

12 Cette référence est fréquemment mentionnée par Ruiz dans ses entretiens; on la trouve par exemple plus d'une dizaine d'années auparavant dans un entretien accordé à la revue Positif ( ${ }^{\circ} 274$, décembre 1983), repris dans Raoul Ruiz. Entretiens, Editions Hoëbeke, Paris, 1999, pp. 45-47.

$13 / d .$, p. 9. 
14 ld., p. 13.

15 Raoul Ruiz, "Fascination et distanciation", dans Poétique du film 2, Editions Dis Voir, Paris, 2006, p. 36.

16 ld., p. 10. multiplier les faits, de les ramifier ou de les perdre en chemin, Ruiz met notamment en crise la notion même d' «action", corrélative de celle de «sujet». Cette désinvolture qui est en quelque sorte une forme paroxystique du plaisir du conteur - ce dernier ayant une conscience aiguë de ceux qui l'ont précédé - induit un rapport particulier au spectateur: celui-ci s'accommode d'une certaine forme d'ennui que Ruiz défend dans son article, inscrivant à cet égard son propre travail dans la lignée de Snow, Wahrhol ou Straub, mais aussi d'Ozu ou Tarkovski14. On peut considérer que cet aspect fait retour dans un autre texte de Ruiz, significativement intitulé "Fascination et distanciation", dans lequel il conteste également la conception hollywoodienne du récit:

"Certains pensent que ce qui peut arriver de mieux quand nous regardons un film est d'être fasciné du début à la fin. Que la distanciation est inutile, ennuyeuse. Il existe une expression qu'utilisent beaucoup de fabricants de films d'Hollywood: quand tu perds le spectateur (lorsque tu cesses de le fasciner), tu le perds pour toujours. Selon ce critère, la distanciation n'est pas seulement inutile, mais dangereuse. Ce n'est pas ce que je pense. J'ai quelques raisons de croire que la distanciation est indispensable, non seulement pour appréhender rationnellement le film [...], mais aussi pour vivre les faits du film dans toute leur complexité. »15

On le voit, Ruiz ne retient pas le modèle de l'immersion continue visant à maximiser les effets destinés à happer le spectateur dans les rets de l'intrigue. Si certains considèrent la distanciation comme «ennuyeuse», il valorise quant à lui précisément ce type d'ennui qui est le garant d'une perception plus complexe des événements. La fascination n'est pas niée, mais inscrite dans une alternance avec des effets ponctuels de distanciation. On pourrait même dire qu'il réside un fort potentiel de fascination dans la façon dont Ruiz met en place cette distanciation, notamment par le biais du foisonnement des faits. Le spectateur n'est pas guidé «du début à la fin", mais confronté à un grand nombre de fragments. En effet, les films de Ruiz se présentent comme discontinus en raison de l'autonomisation qui y est faite de l'unité de la séquence. Arrachées aux principes traditionnels de la concaténation logique et de la vectorisation vers un après (qui est in fine la clôture du récit), ces séquences tendent à flotter hors du temps (ou dans un éternel présent), se donnant comme purs objets de contemplation (et, à n'en pas douter, de "fascination»). Obéissant à son idée selon laquelle "les images qui composent le film déterminent le type de narration qui le structure, et non le contraire »16, Ruiz refuse le primat du Narratif qui caractérise le cinéma classique, bouleversant totalement la manière de fabriquer et de percevoir 
un film. Ainsi l'ancrage temporel d'une scène est-il souvent brouillé - il n'est pas étonnant que le cinéaste se soit attelé à l'adaptation du Temps retrouvé (France/Portugal/Italie, 1998) - par une forme de stase, abandon au pouvoir d'attraction de l'image en tant que tel.

Ce suspens - équivalent dans la distanciation du suspense de la fascination "classique» - résulte fréquemment d'un décalage entre le récit de la voix over et ce qui est visualisé : le narrateur over n'introduit pas l'enchâssement audiovisuel, mais fait porter son récit sur ce qui s'est passé avant lui, ou sur ce qui se passera après. Il nous paraît révélateur que le court métrage Colloque de chien (France, 1977) n'utilise l'image animée que pour des segments descriptifs et intercalaires, les événements proprement dits, répétitifs et cycliques, étant visualisés sous la forme d'un roman-photo. De cette manière, Ruiz tente de soustraire le Narratif à l'inexorable orbite du temps et de faire primer la construction de mondes possibles. Rappelons qu'à ses yeux, le cinéma du direct est "pornographique» en raison de sa prétention à reproduire l'instant présent, c'est-à-dire à nier le caractère représentationnel de la reproduction $\mathbf{1 7}$. Dès lors, on comprend que le tableau vivant suscite son intérêt $\mathbf{1 8}$. Forme mixte entre la vie et la mort qui convient bien à la figuration d'êtres fantomatiques comme ceux qui peuplent ses films, le tableau vivant permet de figer l'avant ou l'après ${ }^{19}$, voire de désigner l'avant et l'après dans la fusion d'un instant (faussement) prégnant qui abolit la chronologie, tandis que l'instant présent et l'action proprement dite, fuyants, échappent à toute visualisation pour être (au mieux) cernés par la parole sur un mode hypothétique. Or, comme le montre L'Hypothèse du tableau volé (France, 1979), l'interprétation demeure soumise à une logique narrative (ou du moins "séquentielle»), puisqu'il s'agit d'isoler des paramètres permettant d'inscrire chaque tableau dans une série (nécessairement incomplète, puisque l'un d'eux aurait été volé). Ce qui distingue les films de Ruiz des conceptions traditionnelles (passablement restrictives, souvent normatives) de la «mise en intrigue» tient à ce que ces dernières exigent, comme l'a relevé Jean-Michel Adam à la suite de Paul Ricœur notamment, une composante "configurante» sous la forme d'une "compréhension dans un acte de jugement qui arrive à tenir [les faits] ensemble au lieu de les voir en séries» 20. A propos de L'Hypothèse du tableau volé, Hervé Gauville parle d'un «non-choix constant» où «les contraires s'affichent, les thèses s'opposent à leur antithèse, mais [où] rien ne se fond dans un ensemble, rien ne se résout en synthèse » $\mathbf{2 1}$. Chez Ruiz, l'éclatement de l'acte de jugement, tour à tour imputé à diverses instances énonciatives (ou formulé à travers des hypothèses divergentes qui coexistent néanmoins), interdit une clôture
17 "Ce qui me choque dans l'art pornographique - j'entends par là aussi bien dans les actualités télévisées - c'est précisément l'effet de direct, c'est la volonté de dire: 'ça se passe en ce moment, là'. A cet égard, les films pornos sont beaucoup moins pornographiques que certains spectacles à la télévision, car ils sont obligés de tricher " (entretien avec Fabrice Revault d'Allones, catalogue du colloque "Peinture-Cinéma", Quimper, 1987, repris dans Christine Buci-Glucksmann et Fabrice Revault d'Allones, Raoul Ruiz, Editions Dis Voir, Paris, 1987 , p. 107). Les connotations pornographiques demeurent d'ailleurs sous-jacentes: le tableau vivant figure dans l'Hypothèse $d u$ tableau volé une cérémonie (à moins que cela ne soit l'inverse, la cérémonie visant à imiter une image fixe) qui ressemble fort à une partie de débauche. Au lieu d'en conserver une trace, la reconstruction qu'est le tableau vivant tend plutôt à occulter l'événement en ajoutant une couche supplémentaire de représentation (à l'image des tableaux du manoir de Généalogies d'un crime où sont organisées les "séances" de tableau vivant: la propriétaire du lieu a fait repeindre son visage sur celui des courtisanes figurées sur les toiles).

18 Voir à ce propos l'article de Valentine Robert dans ce dossier de Décadrages.

19 Dans Généalogies d'un crime, son usage thérapeutique implique de représenter ce qui précède de quelques instants le délit.

20 Louis O. Mink cité par Jean-Michel Adam, Le Récit, PUF, Paris, 1994, p. 93.

21 Hervé Gauville, "Faut-il soutenir L'Hypothèse du tableau volé ?", in Trafic, $n^{\circ}$ 67, automne 2008, pp. 115-116. 
22 Voir Raphaël Baroni, La Tension narrative, Seuil, Paris, 2007.

$23 \mathrm{~A}$ propos de la notion de "diégèse " et de ses liens avec la sémantique des mondes possibles, voir mon article "La , diégèse, dans son acception filmologique. Origine, postérité et productivité d'un concept ", in Cinémas, vol. 19, $n^{\circ} 2-3$, printemps 2009, pp. 218-245.

24 Gérard Genette, Fiction et diction, Paris, Seuil, 2004.

25 Raoul Ruiz, "Supplément au Voyage autour de ma chambre", in Caméra/stylo, n 4 ("Scénario"), septembre 1983, p. 39.

26 André Gaudreault, Du littéraire au filmique. Système du récit, Nota Bene / Armand Colin, Paris, 1999 (1988). à fonction configurationnelle, sapant toute prétention à subsumer les «séries» en un tout. Ses récits travaillent bien plus la «tension narrative" que la dimension téléologique 22.

Chez Ruiz, l'acte configurationnel ne peut s'effectuer dans la mesure où aucun fait n'y est à proprement parler actualisé, les événements évoqués appartenant au registre du possible qui se manifeste selon diverses modalités (rêverie, fantasme, auto-mise en scène, feintise, etc.) et s'inscrit dans un ensemble qui échappe à toute logique hiérarchisante. Plus que comme un récit, c'est comme un monde - comme une "diégèse", pourrait-on dire à la suite des filmologues - que Ruiz envisage chacun de ses films, ou plutôt comme un ensemble de mondes. Les explications qu'il donne consonnent souvent avec l'approche des logiciens - on sait son intérêt pour la philosophie analytique anglo-saxonne (Bertrand Russell, Ludwig Wittgenstein) - qui a également été adoptée par certains théoriciens de la littérature (ou plus généralement de la fiction) comme Umberto Eco ou Thomas Pavel 23. Dans les références qu'il convoque dans ses interviews, Ruiz n'opère jamais le pont entre la philosophie du langage qu'il cite volontiers et les théories de la fiction d'obédience linguistique auxquelles Gérard Genette, par exemple, a travaillé dans Fiction et diction ${ }^{\mathbf{2 4}}$. Il nous semble toutefois que l'œuvre de Ruiz constitue une application passionnante de ce type de réflexions, ses récits se présentant comme une sorte de laboratoire pour mathématicien. Cette conception «mondaine» (ou «modale») du film lui permet d'articuler les niveaux visuel et narratif autour de l'idée d'un changement de "dimension»: "Oui, il y aurait autant de dimensions que d'instances visuelles, voire autant d'espaces que de points de vue; oui, il y aurait autant de mondes que de perspectives du monde»25. En accentuant les contrastes entre l'avant-plan (démesurément grossi par exemple) et l'arrière-plan d'une image - procédé inspiré du style des comics d'Alex Raymond ou de Chester Gould, récurrent dans La Ville des pirates (France/ Portugal, 1983) par exemple -, Ruiz souligne le lieu d'origine d'un regard qui, n’appartenant à aucune instance diégétique, renvoie à la présence $\mathrm{du}$ "monstrateur» (pour reprendre l'expression de Gaudreault) 26. En raison de l'absence d'un point de vue assignable à un personnage du film, il ne s'agit pas tant de «focalisation" au sens de Genette que d'une ouverture, au sein même de l'image, à d'autres mondes susceptibles d'occasionner d'autres récits. Dans un film comme Klimt (Autriche/ France/Allemagne/Angleterre, 2005), l'usage de la surimpression de motifs inspirés des œuvres du peintre éponyme joue un rôle similaire: le palimpseste visuel affecte l'image d'une subjectivité qui, parfois, demeure sans sujet. 
La logique de fragmentation et de démultiplication qui est à l'œuvre chez Ruiz s'applique également à une composante-clé du récit, et du "conflit central» en particulier: il s'agit du personnage. C’est désormais sur cette notion que nous mettrons l'accent, car nous pensons qu'elle emblématise les options singulières de Ruiz quant à la construction de ses récits. Dans l'article "Théorie du conflit central», le cinéaste revendique la possibilité que le personnage n'ait aucun choix à faire, alors que la théorie en question exige que la décision du sujet (qui peut également consister en un refus) soit l'élément déclencheur du récit. Il analyse comme suit le rôle endossé par le personnage dans ce système qu'il récuse:

"La théorie du conflit central produit de la fiction sportive et se propose de nous embarquer pour un voyage dans lequel, prisonniers de la volonté du protagoniste, nous sommes soumis aux différentes étapes du conflit dont le héros est tout à la fois gardien et captif»27.

Ce qui gêne Ruiz dans cette conception hégémonique du récit qui, pour lui, est déterminée par une culture et une idéologie données et ne constitue donc pas un principe immuable, c'est qu'elle vise à «emprisonner» le spectateur - l'isotopie carcérale et hiérarchique révèle dans cette citation combien son cinéma est étranger à une telle visée coercitive - dans le champ des seuls possibles déterminés par la volonté du protagoniste. Les intentions du personnage constituent le principal moteur du récit, provoquant un paradoxe qui consiste à faire de lui simultanément le "gardien» et le "captif» du conflit, dont il motive la présence tout en la subissant. Pour sortir de cette détermination du récit basée sur le personnage (et notamment sur sa caractérisation psychologique), Ruiz se refuse à donner l'illusion que le personnage - et même le narrateur over du film - est celui qui "mène la barque" du voyage (même s'il se plaît, qu'il s'agisse d'un contexte maritime ou non, à "mener en bateau" son spectateur). A la confrontation se substitue la rencontre fortuite chère aux surréalistes (eux-mêmes admirateurs du roman populaire d'aventures) sur laquelle pèse néanmoins le poids du fatum. Les principes ludiques de l'aléatoire qui sous-tendent la construction de ses films transforment le personnage "sujet» en un "objet» manipulé par une instance située à l'extérieur de la diégèse ou au seuil de celle-ci, incarnation évanescente d'un principe structurel. C'est par ce biais que Ruiz entend éviter la mise en place d'un «conflit central» sans renoncer à la forme narrative :

«Une histoire est-elle concevable sans centre ni point de décision? Personnellement, j'ai cherché à travailler avec des histoires, assez abstraites je l'admets, utilisant ce que l'on pourrait appeler un modèle pentaludique, ou pour le dire plus simplement, considérant mes protagonistes comme une 〈troupe〉 de dés » $\mathbf{2 8}$.
27 Raoul Ruiz, "Théorie du conflit central", op. cit., p. 14.

28 /d., p. 19. 
29 Robert McKee, Story: Contenu, structure, genre - les principes de l'écriture d'un scénario, Dixit, Paris, 2001 (1997), p. 101.

30 Syd Field, Comment identifier et résoudre les problèmes du scénario, Dixit, Paris, 2006, p. 136.
En jouant avec ses personnages comme avec des dés qui révèlent une autre face à chaque nouveau lancer et occasionnent de multiples combinaisons, Ruiz s'attaque à l'un des principaux facteurs de la cohérence du récit «classique»: la récurrence et la stabilité de l'actant sujet. Car plus encore que le "conflit central», c'est la construction du personnage qui est au cœur de la majorité des manuels de scénario; en fait, ces deux notions sont envisagées de façon solidaire puisque, comme le postule Robert McKee, "la structure c'est le personnage et le personnage c'est la structure»29. Or les déficiences supposées qui sont énumérées par Syd Field dans son ouvrage de "recettes" intitulé Comment identifier et résoudre les problèmes du scénario semblent constituer un catalogue des pratiques ruiziennes, tant celles-ci s'opposent systématiquement aux préceptes des «script doctors». Syd Field identifie les erreurs suivantes 30 : «le personnage principal est trop passif, trop introverti, on l'oublie» (chez Ruiz, il agit en effet très peu, si ce n'est en tant que conteur, et c'est son introversion qui produit le récit); "tous les personnages se ressemblent» (chez lui, ils tendent à ressembler plus aux autres qu'à eux-mêmes, voire à n'être qu'un seul «individu»); "les personnages secondaires sont plus intéressants et plus forts que le personnage principal» (Ruiz ne respecte aucune hiérarchie en faisant soudain intervenir un protagoniste secondaire qui occupe le devant de la scène); "les relations sont trop vagues, pas assez clairement définies" (l'indécidable prime dans les relations entre les personnages ruiziens); "les dialogues sont trop littéraires, trop élaborés, trop évidents» (Ruiz se plaît à construire des répliques savantes sur la base de citations littéraires, ou à se servir de stéréotypes réitérés afin de souligner la vacuité du sens et la fonction représentationnelle du langage). Ce petit jeu d'opposition entre les normes classiques et la pratique de Ruiz nous permet de souligner la singularité du récit ruizien considéré du point de vue de la construction du personnage. C'est cet aspect en particulier que nous proposons d'approfondir dans la seconde partie de la présente étude, forcément sommaire au vu de l'ampleur de l'œuvre du cinéaste et de la profusion des lignes d'intrigues et des procédés inédits dont fait montre chacun de ses films.

\section{Des personnages multiples: palimpsestes et combinatoires}

Il arrive souvent chez Ruiz que les personnages soient avant tout les porteurs d'histoires qu'ils content à un interlocuteur. La structure qui en découle conjugue la mise en scène du narrateur au sein d'une situation cadre - aux allures parfois très fabriquées, comme dans Les Ames fortes (France/Belgique, 2000) - et des séquences enchâssées donnant lieu à l'audiovisualisation du récit. Dans le cas de narrateurs "homodiégé- 
tiques» (au sens de Genette) ${ }^{31}$, un dédoublement s'opère donc entre le Je narrant et le Je narré, deux niveaux que Ruiz se plaît à mêler au début des Trois Couronnes du matelot en introduisant le personnage d'un étudiant à propos duquel la voix over du marin nous dit "qu'il préférait se nommer à la troisième personne», puis précise qu'il «préférait parler de lui à la première personne dans les moments d'ennui». Cette incertitude quant à l'identité d'un personnage (et du narrateur) est constante chez Ruiz, qui ne cesse de chercher l'Autre dans le Même au gré de variations successives qui constituent la matière même du récit.

L'expression de l'altérité se manifeste parfois à travers l'une des caractéristiques essentielles de l'individualité du conteur: la voix. Prenant à la lettre le sentiment formulé par Ferdinand dans Pierrot le fou (Jean-Luc Godard, France, 1965) - "on devrait avoir l'impression d'être unique, j’ai l'impression d'être plusieurs» (la "machine pour voir» s'étant désolidarisée de la «machine pour entendre») -, Ruiz est l'un des rares cinéastes à recourir fréquemment à des phénomènes de déliaison qui exhibent l'hétérogénéité des matières de l'expression filmique généralement occultée par l'illusion du synchronisme voco-labial. Dans de tels moments, souvent convoqués par le cinéma d'horreur (voir le récent Drag me to Hell de Sam Raimi), la voix proférée est, dans son grain même, fondamentalement extérieure au corps du locuteur visualisé auquel le spectateur est pourtant incité à la rapporter ${ }^{32}$. Cette greffe est une manifestation sur le plan audiovisuel du palimpseste ruizien, qui crée une scission dont j'ai montré les liens qu'elle entretient avec «l'inquiétante étrangeté» freudienne ${ }^{33}$. La dimension surnaturelle (la voix de l'au-delà, produite sans appareil phonatoire) et mécanique (elle résulte de la séparation affichée entre la bande images et la piste sons) participe de la conception que Ruiz se fait de la représentation de spectres:

"J'ai peur des fantômes; c'est tout et c'est très simple. Comment ne pas avoir peur des fantômes, surtout mécaniques? Comment ne pas avoir peur lorsqu'à la télé vous entendez la voix de celui qui vient de mourir?» 34

Ainsi, dans un film fantastique comme La Ville des pirates, la déliaison vocale provoque un effet horrifique lorsque l'enfant, apparition démoniaque qui conduit la servante au meurtre, s'écrie brusquement «Vat'en!» d'une voix masculine adulte, grave et tonitruante. Abolissant momentanément le pouvoir des apparences (l'amant dans un corps d'enfant, la violence insufflée par une voix innocente), ce cri claque comme le surgissement du malin, illusion audiovisuelle - il s'agit d'un emploi détourné du doublage $\mathbf{3 5}$ - qui contrecarre le leurre destiné à l'héroïne au sein de la diégèse. Dépossédé de sa voix, le locuteur est assailli
31 Gérard Genette, Figures III, Seuil, Paris, 1972.

32 A propos du concept de "déliaison vocale", voir mon ouvrage Du bonimenteur à la voixover, Antipodes, Lausanne, 2007, notamment dans un passage consacré au film L'Exorciste (William Friedkin, Etats-Unis, 1973), pp. 414416.

33 Voir ma thèse de doctorat, Les Voix du cinéma. Boniment, voix synchrone, déliaison et voix-over, Université de Lausanne, mars 2006, "L'inquiétante étrangeté de la voix déliée", pp. 312-324.

34 "Entretien", dans Christine Buci-Glucksmann et Fabrice Revault d'Allones, Raoul Ruiz, op. cit., p. 100 (nous soulignons).

35 René Clair, dont j'ai souligné ci-dessus (voir note 1) la parenté que son cinéma entretient avec celui de Ruiz, a relevé à propos de cette pratique de post-production: "Du doublage, Jean Renoir dit fort justement que ceux qui s'en rendent coupables, s'ils avaient vécu à une époque raisonnable comme le Moyen Age, auraient été brûlés en place publique pour avoir donné à un corps une voix qui ne leur appartient pas, ce qui ressemble fort à un crime de sorcellerie " (Cinéma d'hier, cinéma d'aujourd'hui, Gallimard, Paris, 1970, p. 201). Nous reviendrons sur ces questions dans Décadrages à travers un dossier consacré au doublage, prévu pour 2011. 


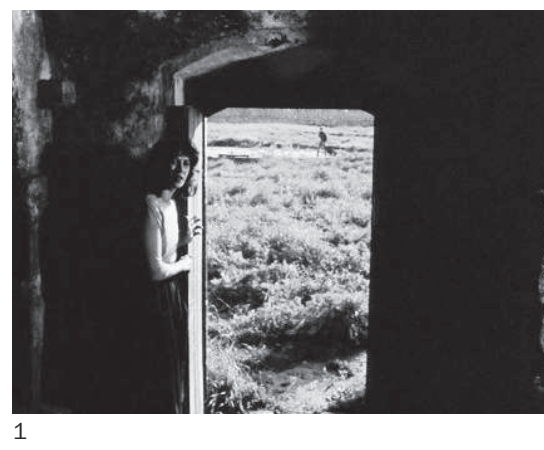

36 Cette intrusion toute ruizienne d'une forte discontinuité sémantique au sein d'une pratique (ici de montage) continue conduit dans le dernier contrechamp à un passage du noir et blanc à un plan en couleurs. par la présence de l'autre qui s'exprime à travers lui : cet usage vocal s'inscrit dans la thématique de l'éclatement de l'individu qui traverse toute l'œuvre de Ruiz, où elle est associée à l'angoisse d'une identité impossible, car toujours soumise à l'indécision du monde dont le polymorphisme des personnages est l'une des manifestations. Dans La Ville des pirates, chacun des deux protagonistes principaux endosse tour à tour des personnalités multiples: la captive du château se contente d'abord, située au seuil de la diégèse, de commenter à l'intention du spectateur les diverses incarnations mimées par le personnage à l'arrière-plan (fig. 1), avant d'entrer littéralement dans son jeu - et cela également au sein d'une même discussion, comme dans une séquence en champ/contrechamp (fig. 2-3) où chacun assigne successivement à l'autre un rôle différent en changeant inopinément de personnalité afin d'émettre un jugement sur le personnage précédent ${ }^{36}$. Enfin, cette logique permutative aboutit, à l'issue d'un long et violent baiser sur la bouche, à un transfert de personne, la femme se mettant à parler, à la suite de cette étreinte forcée, avec la voix de son amant. Ce genre de procédés qui évoquent le burlesque d'un Tex Avery - mais qui, déplacés dans un film à acteurs, relèvent du cauchemardesque - irréalise totalement le monde représenté. Dans Les Trois Couronnes $d u$ matelot, alors que le "héros ", qui est aussi le narrateur over, dit à l'un des matelots surnommé «l'imitateur» - on devrait plutôt parler de "ventriloque» - qu'il l'a déjà vu quelque part (ce qu'atteste une séquence préalable, dans laquelle nous l'avons vu se jeter à l'eau), celui-ci lui répond: "Non, ce n'est pas moi, c'est l'autre. Mais c'est la même chose, nous sommes tous pareils.» Cette conversation se poursuit ensuite de façon encore plus étrange car, lorsque le matelot raconte l'histoire de «l'autre» qui l'a trahi (mais serait-ce le même, c'est-à-dire son interlocuteur?), deux voix se superposent, comme si l'acteur qui s'exprime en voix in était simultanément doublé par un fantôme à la voix gutturale. Un tel palimpseste sonore participe à nouveau d'un jeu sur le franchissement
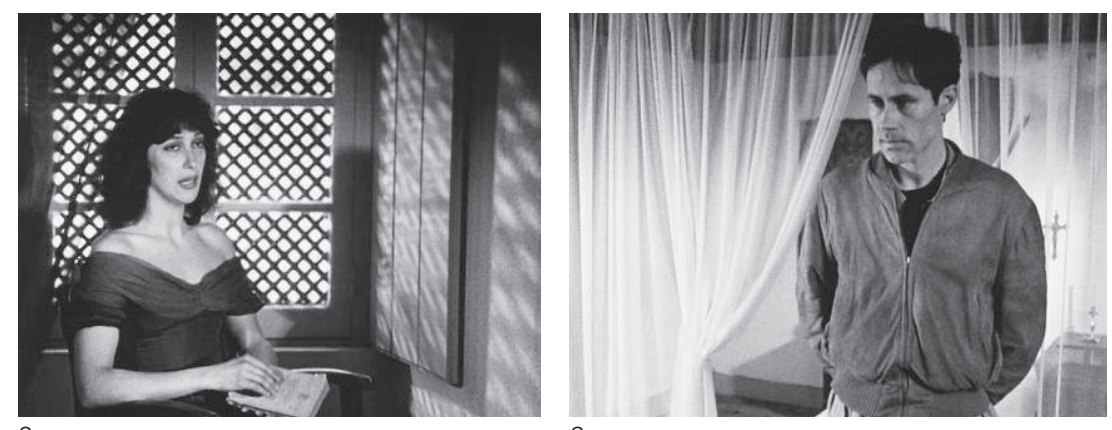

3 


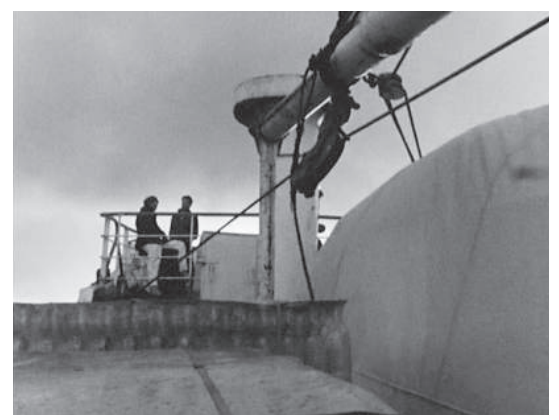

4

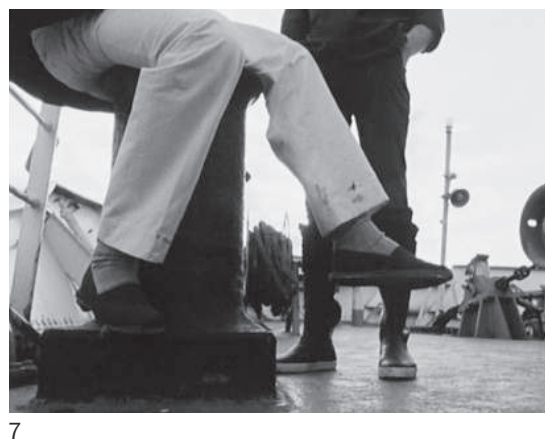

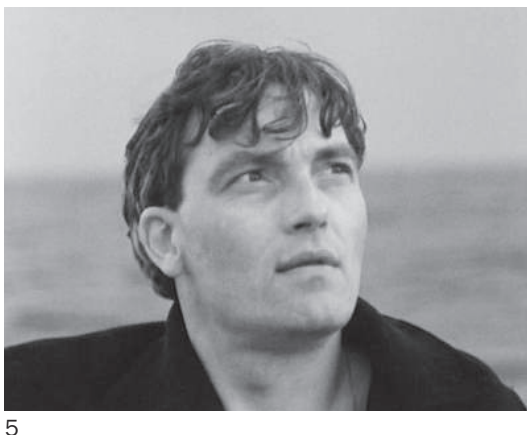

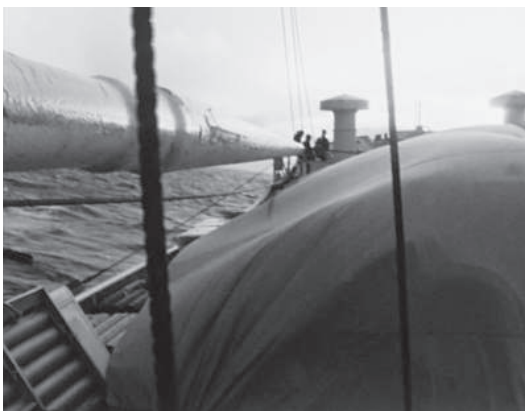

8

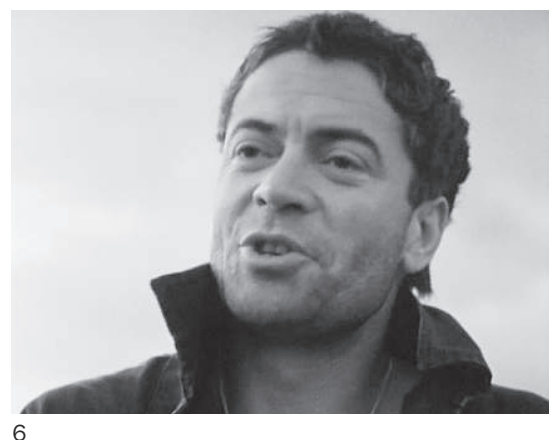

6

d'une autre «dimension", car Ruiz exploite le caractère perméable de la frontière entre les voix in et over ${ }^{37}$, démarche favorisée par sa tendance, dans des séquences de dialogue, à reléguer hors-champ les locuteurs en privilégiant des plans qui rompent la continuité des raccords en champ/ contrechamp ou à brouiller les repères spatiaux en nous éloignant soudainement des personnages - à l'instar de la séquence de «l'imitateur» des Trois Couronnes du matelot évoquée ci-dessus (fig. 4-8) - voire, sur le mode de l'ouverture de Muriel (Alain Resnais, France, 1963), en introduisant de nombreux inserts de détail sur des objets environnants.

Les voix déliées constituent l'un des indices de l'instabilité du personnage ruizien, qui est constamment mis à mal dans son intégrité ontologique. Car, à l'instar des personnages de certains films de David Lynch (Lost Highrway, Mulholland Drive, Inland Empire) ${ }^{38}$, ceux de Ruiz mutent pour en devenir d'autres. Toutefois, alors que ces phénomènes de transformation ou de permutation œuvrent chez Lynch (où ils sont ponctuels) à l'établissement de pivots narratifs - il s'agit de véritables gouffres dans lesquels basculent le spectateur -, ils s'opèrent chez Ruiz sans dramatisation aucune. En effet, en dépit de l'étrangeté des événements, nul basculement ne se produit: les mutations identitaires sont d'autant plus troublantes que Ruiz souligne l'indifférence du monde au destin

37 Ainsi dans L'Hypothèse du tableau volé, le collectionneur qui s'exprime en voix in interagit avec le discours over, rompant l'extranéité qui régit conventionnellement les rapports entre les niveaux diégétique et extra-diégétique.

38 Voir à ce propos mon article "L'éclatement du personnage chez Lynch. Les imondes possibles > dans Lost Highway et Mulholland Drive" in Décadrages, n 4-5, pp. 48-61. 
39 "Entretien", dans Christine Buci-Glucksmann et Fabrice Revault d'Allones, Raoul Ruiz, op. cit., p. 84. Voir également l'entretien réalisé pour le présent dossier (p. 83), dans lequel Ruiz signale l'influence qu'a exercée son travail pour la télévision sur ses films de fiction - ainsi précise-t-il à propos de la production télévisuelle Petit manuel d'histoire de France (France, 1979) que le personnage de Napoléon y a "quatorze visages", tandis qu'à l'inverse un même acteur peut se "transforme[r] tout à coup en prêtre des templiers, puis en pape.".

40 Raoul Ruiz, "Fascination et distanciation", op. cit., p. 38. humain. Au changement de monde sursignifié par la «boîte magique» de Mulholland Drive (autre film marqué par la déliaison vocale) correspond chez Ruiz le glissement «naturel» du Même vers l'Autre. C'est que chez Ruiz, ces mutations prennent place dans un ensemble dominé par la discontinuité. On retrouve là l'inspiration des séries B évoquées dans «La théorie du conflit central», ainsi que dans l'entretien suivant, éclairant quant à l'attirance de Ruiz pour des personnages multiples :

"Fabrice Revault d'Allones: Au cinéma, vous retrouvez le côté <feuilleton', avec d'étranges métamorphoses, une continuité discontinue, des glissements...

Raoul Ruiz: Il y avait Vincent Price ou Robert Taylor qui jouaient beaucoup de rôles, et comme on projetait plusieurs films différents par soir dans ces salles populaires du Chili, un même acteur jouait l'espion soviétique dans un film, Ivanohé dans un autre, se transformait en cow-boy qui mourait avant de réapparaître en avocat boiteux... Bref, trois ou quatre personnages pour un même acteur.» $\mathbf{3 9}$

Comme les films de Ruiz aux ramifications variées et aux finales à rallonge compriment en quelque sorte plusieurs films en un (souvent en faisant cohabiter plusieurs genres), il n'est pas surprenant que les personnages subissent de telles modifications. Le spectateur en ressort avec l'impression d'avoir vu plusieurs "petits» films en cascade, l'effet d'ensemble oscillant entre l'outrance «baroque» et la posture parodique. Cette hétérogénéité ne concerne cependant pas le seul niveau de la "séquence narrative», mais aussi la discontinuité foncière du cinématographe :

«N'est-il pas vrai que dans une séquence de n'importe quel film, aussi banal soit-il, l'on a habituellement un personnage $\mathrm{X}$ dans une scène au premier plan, que l'on voit immédiatement après en plan général? Y a-t-il quelque chose qui interdise d'imaginer que la seconde prise montre le double du personnage que nous avons déjà vu au premier plan? Et ensuite que, chaque fois que nous le voyons dans la séquence $b$ et $\mathrm{c}$ et $\mathrm{d}$, nous verrons en réalité les multiples doubles du premier personnage ? » $\mathbf{4 0}$

Faisant fi de tout principe de raccord, Ruiz dénaturalise la lecture linéarisante traditionnelle et propose d'exhiber chaque saute, d'y voir le seuil d'un autre monde. Le changement de plan s'accompagne, en dépit de la permanence de l'acteur, d'un changement de personnage. Cette logique est celle du truc par substitution que Méliès effectuait certes non au niveau du montage, mais au niveau du tournage. Cependant, dans Les Trois Couronnes $d u$ matelot, Ruiz va jusqu'à introduire de telles variations au sein d'un même plan-séquence: le marin qui croit retourner chez ses parents est abandonné hors-champ par un mouvement d'appareil 
qui, semblant peu se soucier de lui, balaie la façade de l'édifice, puis est rattrapé par le personnage qui traverse le champ pour sortir sur la gauche (fig. 9-17). Comme ce dernier réapparaît ensuite en entrant par la gauche, la logique du raccord de direction est maintenue, mais elle est brisée un peu plus tard, lorsque le matelot, sorti par la droite, réapparaît à gauche pour se précipiter dans la même direction. Du point de vue de la cohérence de la représentation spatiale, ce personnage ne peut être le même que le précédent: il est l'un de ses avatars qui effectue une action similaire, mais avec une issue différente.

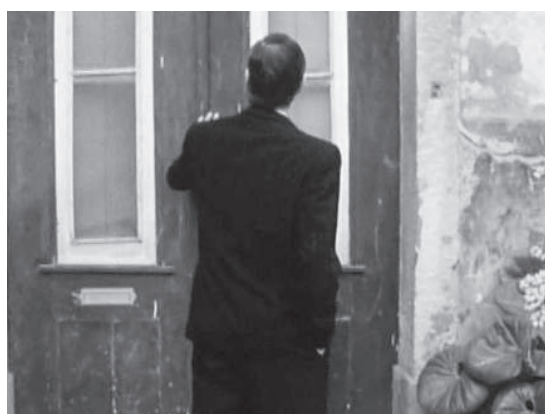

$$
9
$$

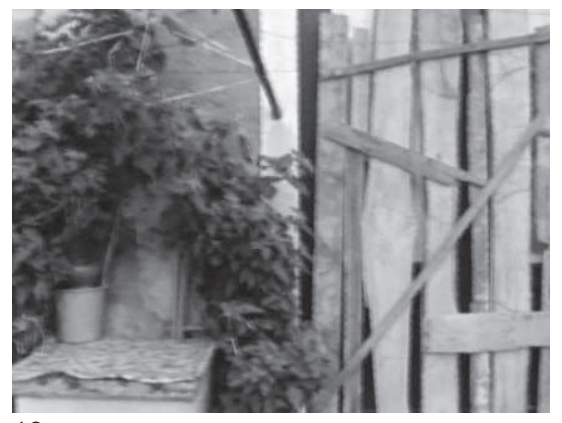

12

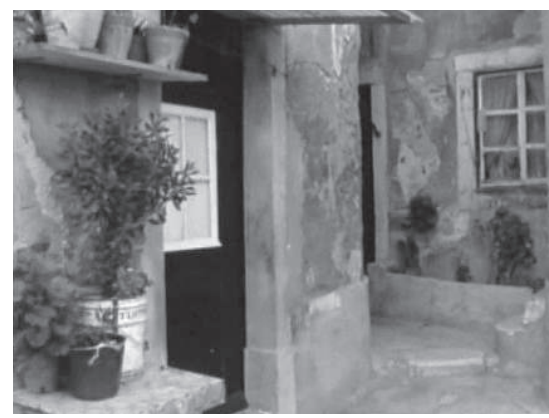

15

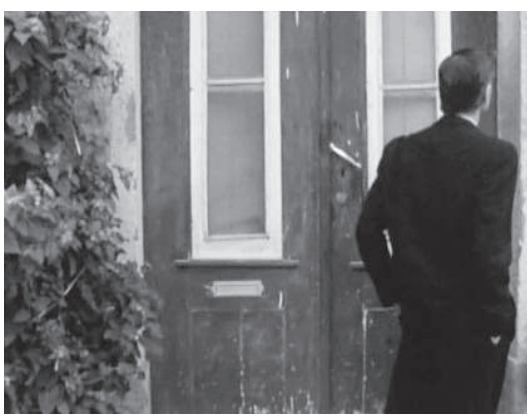

10

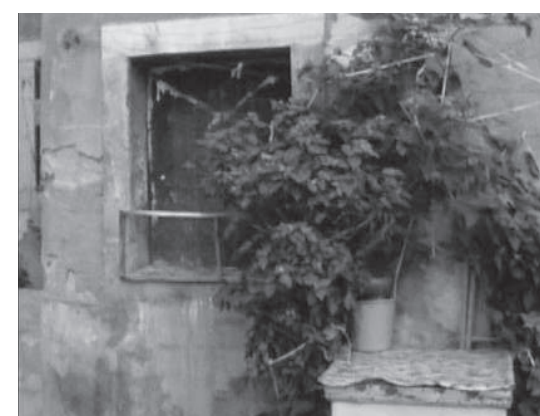

13

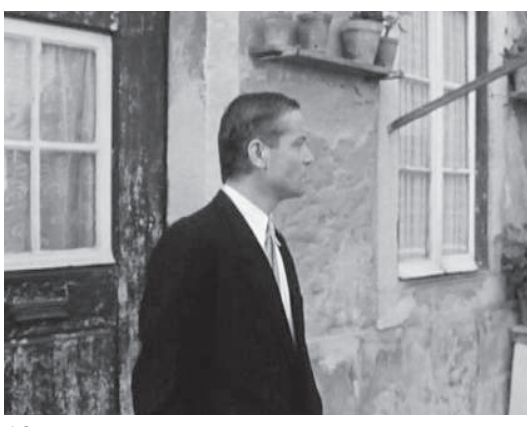

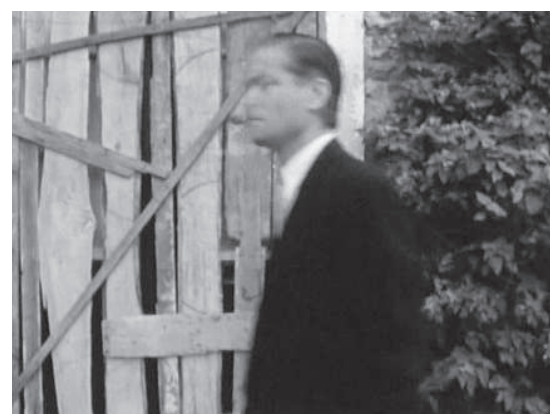

11

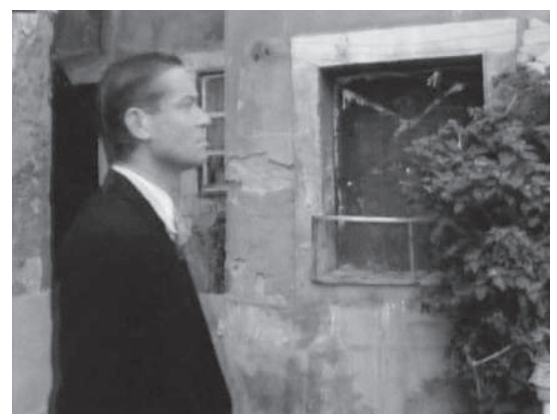

14

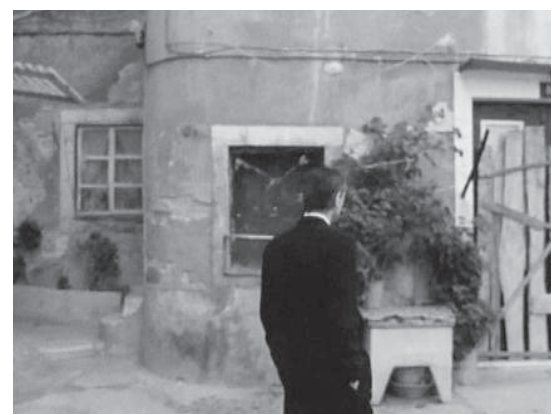

17 
41 Carl Therrien, "Le cinéma sous l'emprise du jeu", dans R. Audet et alii, Jeux et enjeux de la narrativité dans les pratiques contemporaines (Arts visuels, cinéma, littérature), Editions Dis Voir, Paris, 2006, p. 98.

42 En juxtaposant différentes prises, les films de Ruiz semblent parfois intégrer leur propre making of.
Chez Ruiz, le réel se bombe ou se déchire sous la multiplication des possibles pour une même action donnée. Rappelons-le, le cinéaste dit considérer ses galeries de personnages comme des «troupes de dés» (voir supra). Se référant à Trois Vies et une seule mort (France/Portugal, 1995) (ainsi qu'à Smoking/No Smoking (France, 1993) de Resnais par exemple) et rappelant que, dans Le Jeu de l'oie (France, 1979), Ruiz filme littéralement un coup de dé qui fait "passer le protagoniste à une échelle supérieure", Carl Therrien rapproche implicitement de telles versions alternatives du contexte contemporain des jeux vidéo $\mathbf{4 1}$. Il faut remarquer toutefois qu'il s'agit chez Ruiz d'une véritable conception du monde, du récit et du travail avec les acteurs $\mathbf{4 2}$ qui n'a pas attendu la généralisation de l'informatique pour voir le jour (d'ailleurs le modèle narratif du cinéma dominant vilipendé par Ruiz n'en a pas pour autant été modifié, sinon dans quelques rares cas).

De par son seul titre, Trois Vies et une seule mort synthétise les traits du cinéma de Ruiz que nous avons essayé d'esquisser ici, et constitue en quelque sorte le discours de la méthode de l'élaboration ruizienne du personnage. Dans ce film, l'élément fixe n'est pas l'un des personnages principaux que nous suivons tour à tour, mais l'acteur Marcello Mastroianni qui les interprète tous. Ce cas de schizophrénie n'occasionne aucune psychologisation, il est une donnée de base qui régule les bifurcations narratives indépendamment de toute révélation de l'intériorité du personnage. Affichée, la mécanicité du récit ne procède pas, comme dans la narration classique, de la caractérisation du personnage: les traits qui le définissent n'évoluent pas mais demeurent inféodés aux rôles stéréotypés endossés par ses diverses personnalités. Le protagoniste ne choisit pas de devenir un autre, il est soudain gagné par un accès qui le conduit machinalement à le faire. La transformation pavlovienne en majordome qui s'opère à chaque tintement d'une clochette souligne l'automaticité de ce procédé: le personnage fait preuve d'obéissance, se plie à la structure du récit plus qu'il ne contribue à la forger. Il n'a aucune conscience de sa personnalité multiple. Même si, de façon assez peu vraisemblable, les diverses personnes sises dans le même corps occupent un espace-temps similaire, nous avons l'impression de changer de film à chaque mutation amnésique. De plus, comme souvent chez Ruiz lorsque certaines actions excèdent le cadre réaliste, les personnages périphériques ne s'étonnent guère du "caractère" fragmentaire de la facette $\mathrm{du}$ personnage avec laquelle ils interagissent; comme le spectateur du film, ils acceptent, en tant que spectateurs de la vie du "héros", le faible degré de crédibilité des explications qu'il leur donne. Ainsi, après vingt ans d'une absence mystérieuse, l'un des personnages renoue en toute 


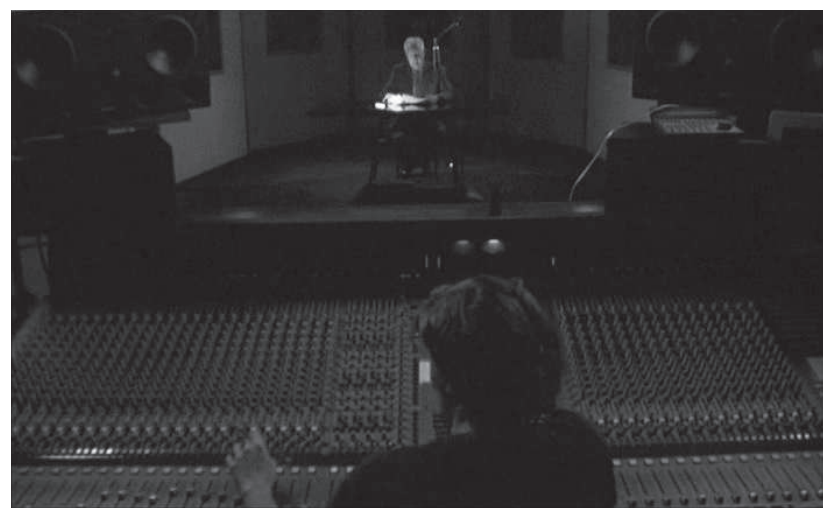

18

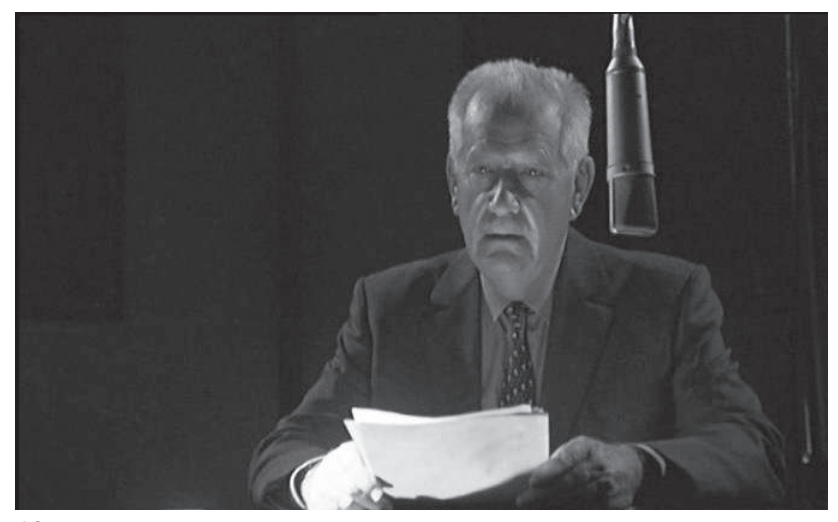

19

quotidienneté avec son ex-épouse, se risquant même à dire que le bébé qu'elle a eu d'un autre et qu'il voit pour la première fois a pris du poids, comme s'il n'avait jamais quitté son foyer. Lors du regard silencieux que s'échange le couple après cette réplique, l'épouse ne manifeste aucune stupéfaction, mais un amusement et une connivence qui caractérisent également le "contrat de lecture» auquel nous invite le film.

Quant à la voix over qui nous accompagne dans le dédale de ce récit, elle ne fournit aucun éclaircissement relatif aux motivations du personnage, se contentant de présenter objectivement les faits tout en créant une proximité complice avec le public. Cette voix, précisons-le, n’est pas toujours over: le narrateur, interprété par Pierre Bellemare, est parfois visualisé alors qu'il lit le texte devant un micro, dans un studio de radio (voir les deux premiers plans du film, fig. 18-19). Dans ces intermèdes, il souligne la dimension épisodique du film en introduisant chaque partie comme une histoire différente (alors que le personnage polymorphe est, lui, "constant»). En raison de sa longue carrière d'animateur radio et TV et de sa réputation de conteur d'histoires qui sortent de l'ordinaire - il en a d'ailleurs fait paraître beaucoup dans des recueils qui furent des best-sellers -, Bellemare représente pour le spectateur français la figure - et la voix! - par excellence du conteur populaire. On le sait, l'animateur aime souligner le caractère à la fois extraordinaire et véridique de ses récits. Sa manière de procéder qui consiste à s'inspirer de faits divers sensationnels pour en souligner l'étrangeté correspond bien au "réalisme» ruizien - «il s'agit de cas bien réels, si réels qu'ils ont eu lieu non pas qu'une fois, mais plusieurs ", déclare le narrateur -, tandis que la masse de récits qu'il s'est appropriée - il en aurait aujourd'hui plus de 7000 au co(mp/n)teur $\mathbf{4 3}$ - rejoint l'inclination du cinéaste pour la prolifération (qui implique chez lui la compilation de plusieurs histoires

43 Dans un entretien récent, Bellemare déclare: "Grâce aux Dossiers extraordinaires que j'ai présentés sur Europe 1 pendant plus de quatorze ans, j'ai rassemblé 4000 histoires vraies; maintenant, j'en ai 7000 au compteur. A l'époque, l'ordinateur n'existait pas, je les tapais à la machine. Mais il y en avait un paquet, alors j'ai dû les rassembler dans une petite maison réservée à ces archives. Avec mon équipe de vingt-trois documentalistes, je les enrichis tout le temps. Nous collectionnons avant tout des coupures de presse. A chaque livre, je rassemble des histoires autour d'un thème, comme les disparitions, les vengeances ou encore les chasses à I'homme." (Anne-Catherine Renaud, "Pierre Bellemare se frotte aux Experts ", Le Matin, 30 mai 2009). 
44 Voir www.lesinrocks.com/cine/cinemaarticle/article/trois-vies-et-une-seule-mort-2. en une seule). Via l'apparition de Bellemare à l'écran - dont les histoires radiophoniques furent pour Ruiz, selon ses propres dires, l'un des premiers contacts avec la culture française $\mathbf{4 4}$-, le cinéaste exhibe sous une forme incarnée le pouvoir démiurgique qu'exerce dans tous ses films le narrateur (ou ses avatars diégétiques, comme ici la figure de l'enfant) sur ses créatures. En dépit du refus de Ruiz d'adopter le "système de crédibilité» dominant, le spectateur est emporté dans les développements ludiques des diverses histoires de ce film reliées de façon sous-jacente et souvent énigmatique par un tissu de motifs récurrents. Il adhère ainsi à une injonction paradoxale du narrateur qui nous semble synthétiser la constante oscillation du cinéma de Raoul Ruiz entre la fascination et la distanciation : comme le dit Bellemare dans Trois Vies et une seule mort, "Suspendons notre crédulité, faisons appel à notre réserve toujours insuffisante d'ironie et de scepticisme, car ces histoires sont contagieuses..." 